\title{
Eventos extremos de lluvia y riesgo hidroclimático en zona de la mata - Pernambuco / Brasil.
}

\author{
Joselma Araújo de Lucena ${ }^{1}$, joselma.geografia@gmail.com; Lucas Suassuna de A. Wanderley ${ }^{1}$; \\ Rodrigo Ranulpho'; João Vitaliano Carvalho Rocha'; Ranyére Silva Nóbrega ${ }^{2}$
}

\section{RESUMEN}

Las fuertes lluvias son fenómenos naturales, pero cuando están asociados al paisaje urbano se convierten en eventos peligrosos, contribuyendo al empeoramiento de las condiciones de vida de la población en la ciudad. En Brasil, los escenarios de degradación del medio ambiente, sufren influencia directa de las desigualdades sociales y de la infraestructura deficiente, como también de la ocupación de los espacios inapropiados, que resultan en el aumento de las vulnerabilidades y de los riesgos. Este artículo tiene como objetivo analizar la ocurrencia de eventos extremos causados por las lluvias en la mesorregión Zona da Mata, en Pernambuco, Brasil, específicamente, en los municipios de Barreiros y Palmares. Además, se investigó las condiciones de riesgo que pudieron contribuir con desastres naturales en el área de estudio. El análisis fue realizado con base, en las estadísticas descriptivas de las series temporales de precipitación, seguido del cálculo del índice de anomalías de lluvia (IAC, en portugués es Índice de Anomalia de Chuva) y análisis de imágenes de satélite para caracterizar el modelo de las lluvias y la dinámica atmosférica, asociando los resultados en el período de ocurrencia de eventos de lluvias fuertes que se concentró en un corto período de tiempo. El principal evento pluviométrico de gran magnitud en los municipios fue en junio de 2010, causado por la Onda de Este que contribuyó al desborde del cauce Una y Mundaú, e inundaciones en varias ciudades de la cuenca.

Palabras clave: Lluvia, Evento Extremo, Inundaciones.

\section{Events extreme rainfall and hidroclimático risk in Pernambuco / Brazil}

\begin{abstract}
Intense rains are natural events associated with urban peculiarities that can become sources of danger, worsening the population's living conditions in the city. In Brazil, the scenarios of environmental degradation resulting from social inequalities and poor infrastructure, coupled with the occupation of inappropriate spaces highlight risks and vulnerabilities that often coincide with urban river environments. This article aims to analyze the occurrence of extreme events caused by rainfall in mesoregion Zona da Mata in Pernambuco, Brazil, specifically, the municipalities of Barreiros and Palmares. Furthermore, the risk conditions that led or may lead to disaster areas were studied. The analysis was carried out, firstly by analyzing the descriptive statistics of the time series of precipitation and later the Rain Anomaly Index (RAI) was calculated. Satellite image analysis was then utilized to characterize the pattern of rain and atmospheric dynamics associated with the period of occurrence of selected rainfall events; heavy rainfall concentrated in a short period of time. The main rainfall event of great magnitude for municipalities occurred in June 2010 caused by Eastern wave disturbance, which led to the flooding of rivers and flooding in several cities in the basin of Una and Mundau rivers.
\end{abstract}

Keywords: Precipitation, Extreme event, Flood.

Recibido el 30 de mayo de 2016; aceptado el 28 de junio de 2016.

\footnotetext{
1 Programa de Pós-graduação em Geografia. Universidade Federal de Pernambuco. Av. Prof. Moraes Rego, 1235-Cidade Universitária, Recife - PE - Brasil. CEP: 50670-901.

2 Departamento de Geografía, Universidade Federal de Pernambuco, Brasil.
} 


\section{INTRODUCCIÓN}

Actualmente los eventos naturales extremos relacionados con el clima son cada vez más frecuentes en la vida cotidiana, muchas veces causa de impactos en la sociedad, especialmente para las poblaciones de los centros urbanos. Los eventos naturales extremos son responsables de importantes daños y pérdidas sociales, económicas y ambientales, resultando en desastres naturales, que a su vez han tenido una creciente ocurrencia, generando impactos cada vez más intensos asociados entre otras causas al cambio climático global (IPCC 2014).

Se ha observado gran interés de la comunidad científica, planificadores y gestores territoriales por el estudio de la frecuencia e intensidad de los fenómenos meteorológicos extremos (GIRAO 2012). Bajo el paradigma de la relación sociedadambiente urbano, en razón de la cual se establece el espacio urbano. Hay consenso en afirmar que la acción humana denota de esta relación nuevos procesos cambiantes y nuevas estructuras, sobre las cuales constituyen una influencia al sistema natural, donde se generan respuestas no pasivas del sistema a las acciones de la sociedad (SILVEIRA \& SARTORI 2010).

Bajo esta perspectiva, el espacio urbano es la expresión concreta relevante de los nuevos escenarios producidos por el hombre en el paisaje natural. Esta segunda naturaleza, la urbana, combinada con el mal uso del espacio y la ocupación desordenada del territorio atribuyen con el retroceso de la vegetación, producción de residuos, al desagüe de las aguas residuales, entre otros factores. Los resultados de los cambios en los procesos naturales implican desequilibrios ambientales en los ecosistemas. En el ecosistema urbano, esta naturaleza ha humanizado la ciudad y su desarrollo es objeto de interés para el estudio de las nuevas situaciones y procesos que le son propias (MENDONÇA 2010).
A pesar de no existir en Brasil registros de fenómenos como terremotos, erupciones volcánicas y huracanes observados en las últimas décadas, se han registrado muchos eventos de deslizamientos de tierra e inundaciones que causan daños y pérdidas significativas, incluyendo las pérdidas de vidas humanas (TOMINAGA et al. 2009). En paralelo al aumento de la intensidad de los fenómenos naturales, el creciente proceso de urbanización que consecuentemente contribuyó con el crecimiento de las ciudades, no siempre se da de manera ordenada y planificada. La desigualdad social de los centros urbanos se refleja en la ocupación de zonas no aptas, lo que resulta en el aumento del peligro y riesgo a los desastres naturales (TOMINAGA et al. 2009).

Según CASTRO et al. (2005), el riesgo es una categoría de análisis asociado a la incertidumbre, exposición al riesgo y las pérdidas materiales, económicas y humanas debido a los procesos de orden natural. En el espacio urbano las situaciones de riesgo derivadas de desastres naturales abarcan una serie de eventos de variadas dimensiones, de los cuales, las inundaciones son más recurrentes. JACOBI (2004) señala que en general la población con los ingresos más bajos son las más expuestas al riesgo. Cuando ocurren inundaciones, las personas que viven cerca del cauce de los ríos, se vuelven vulnerables al fenómeno, pero las de más bajo ingreso financiero son las que estarán más expuestas.

La convivencia con el riesgo que cada ambiente proporciona, es percibida como un peligro inminente de un desastre real. Las inundaciones, son ejemplos de los riesgos a los que un individuo o una comunidad está expuesta, y son resultado de la asociación entre los peligros naturales y los riesgos derivados de los procesos agravados por la ocupación de espacios susceptibles al riesgos (MONTEIRO 1996; VEYRET 2007). 
En la percepción de MOURA \& SILVA (2008) los desastres no son naturales, sino que surge de la acción humana. Por lo tanto, la población urbana convive con factores ambientales, pero los daños ambientales resultan de causas físicas (geológicos, climáticos, etc.) y la acción humana. El mal uso y ocupación desordenada del suelo por la población evidencia las cuestiones relacionadas con los impactos de los fenómenos naturales extremos.

De acuerdo con CIDADE (2013), la dinámica del riesgo en sus diferentes grados está presente en ciudades de todos los continentes, debido a la intensa expansión urbana y el uso inapropiado del territorio, lo que intensifica las amenazas e impactos generado por los eventos naturales extremos. Estos tipos de problemas son recurrentes en América Latina y Brasil.

En esta perspectiva, la ocurrencia de eventos extremos de lluvia, causadores de inundaciones han sido tema de varios estudios científicos y debates sobre el aumento de la incidencia en las últimas décadas (SILVEIRA \& SARTORI 2010; SANTOS et al. 2012; ZAMPARONI 2012).

MARENGO (2008) señala que en el Este de la región Nordeste, que corresponde a la Costa, Zona da Mata y Agreste, la temporada de lluvias se produce entre abril y agosto, y las lluvias son producidas principalmente por el contraste entre la temperatura del mar a lo largo de la costa y de la temperatura del continente. Los sistemas atmosféricos responsables por la precipitación del Noreste, la Zona de Convergencia Intertropical (ZCIT) y sobre todo, las Ondas de Este durante el otoño y el invierno.

Las Ondas de Este se asocian con máximas de precipitación en el período de mayo a julio en el sector oriental del Noreste, la Costa, Zona da Mata y Agreste. En el continente Sudamericano las Ondas de Este son poco conocidas, aunque una acción clave de la convección en la mayoría de los eventos de mediana escala del océano. Las Ondas de Este de África son las más conocidas, y se extienden desde África Occidental hasta el Océano Atlántico tropical, con una máxima actividad en el invierno austral. Estas perturbaciones modulan la convección y por lo tanto la precipitación.

En Brasil los primeros estudios sobre las Ondas de Este fueron realizados por NEIVA (1975) y YAMAZAKI (1977), e identificaron la presencia de los sistemas en el Atlántico Sur, los que se propagan hacia el Noreste de Brasil. MOLLION \& BERNARDO (2002) propusieron que los procesos de formación de las Ondas del Este están relacionados a las penetraciones de sistemas frontales provenientes de los dos hemisferios y de sistemas convectivos mesoescala originados de la región Zona de Convergencia Intertropical (ZCIT) y de la costa de África. BARBOSA (2007) estudió el comportamiento de las Ondas del Este entre 1984 y 1998, concluyendo que las ondas se relacionan con la parte superior del $60 \%$ de las perturbaciones convectivas y que se propagan a más de $450 \mathrm{~km}$ tierra adentro.

La región sur del Zona da Mata pernambucana fue afectada por un evento de lluvia extrema en el período comprendido entre el 16 y el 19 de Junio de 2010, cuando las cuencas de los ríos Una y Mundaú tenían sus niveles históricos del cauce superados debido a un evento que alcanzó casi todas las áreas de las cuencas superiores, causando un rápido aumento de agua de ambos ríos y el consiguiente desbordamiento del cauce, con las inundaciones en las zonas urbanas y en los municipios Palmares y Barreiros.

En esta perspectiva, este estudio tiene como objetivo analizar la ocurrencia del evento de lluvia extrema ocurrido en Junio de 2010, en los municipios de Barreiros y Palmares / PE, que se correlaciona con los sistemas atmosféricos que causaron las lluvias y comprobar los impactos sociales de las 
inundaciones en estas ciudades. Además, se investigó las condiciones de riesgo hidroclimático que han llevado o pueden conducir a desastres naturales en las zonas estudiadas.

\section{MATERIAL Y MÉTODOS}

El área de estudio son los municipios de Barreiros y Palmares, ubicadas en la mesorregión de la Zona da Mata Pernambucana y la micro región de Zona de La Mata Sur, a unos 100 kilómetros de Recife, la capital del estado de Pernambuco. En 2010, Barreiros poseía población de 40.732 habitantes y Palmares 59.526 habitantes (INSTITUTO BRASILEIRO DE GEOGRAFIA E ESTATÍSTICA 2014); ambas ciudades en la cuenca del río Una. El clima de la región es cálido y húmedo, con temperatura media que oscila entre $22^{\circ} \mathrm{C}$ y $25^{\circ} \mathrm{C}$ durante todo el año y totales anuales de precipitaciones superiores a $1.000 \mathrm{~mm}$. El período de lluvias intensas dura aproximadamente seis meses, a partir de marzo y se extiende hasta Julio o Agosto.

El río Una (Fig.1) nace en la ciudad de Capoeiras, en el estado de Pernambuco, a 900 m.s.n.m. y posee aproximadamente $255 \mathrm{~km}$ de largo hasta el Océano Atlántico. La cuenca se encuentra en el extremo sur de la costa de Pernambuco $\left(8^{\circ} 17^{\prime} 14^{\prime \prime} \mathrm{S}\right.$ $\left.8^{\circ} 55^{\prime} 28^{\prime \prime} \mathrm{S}-35^{\circ} 07^{\prime} 48^{\prime \prime} \mathrm{W}-36^{\circ} 42^{\prime} 10^{\prime \prime} \mathrm{W}\right)$ y tiene una superficie total de $6274 \mathrm{~km} 2$ (MACHADO et al. 2012).

Los datos de precipitación usados en el estudio se obtuvieron del Sistema del Control Agrometeorológico (Agritempo) y Agencia Pernambucana de la Agua y Clima (APAC). Para la ciudad de Barreiros se utilizó la serie histórica del período 1963-2011 y para Palmares el período 19602011. La tabulación de los datos de lluvia

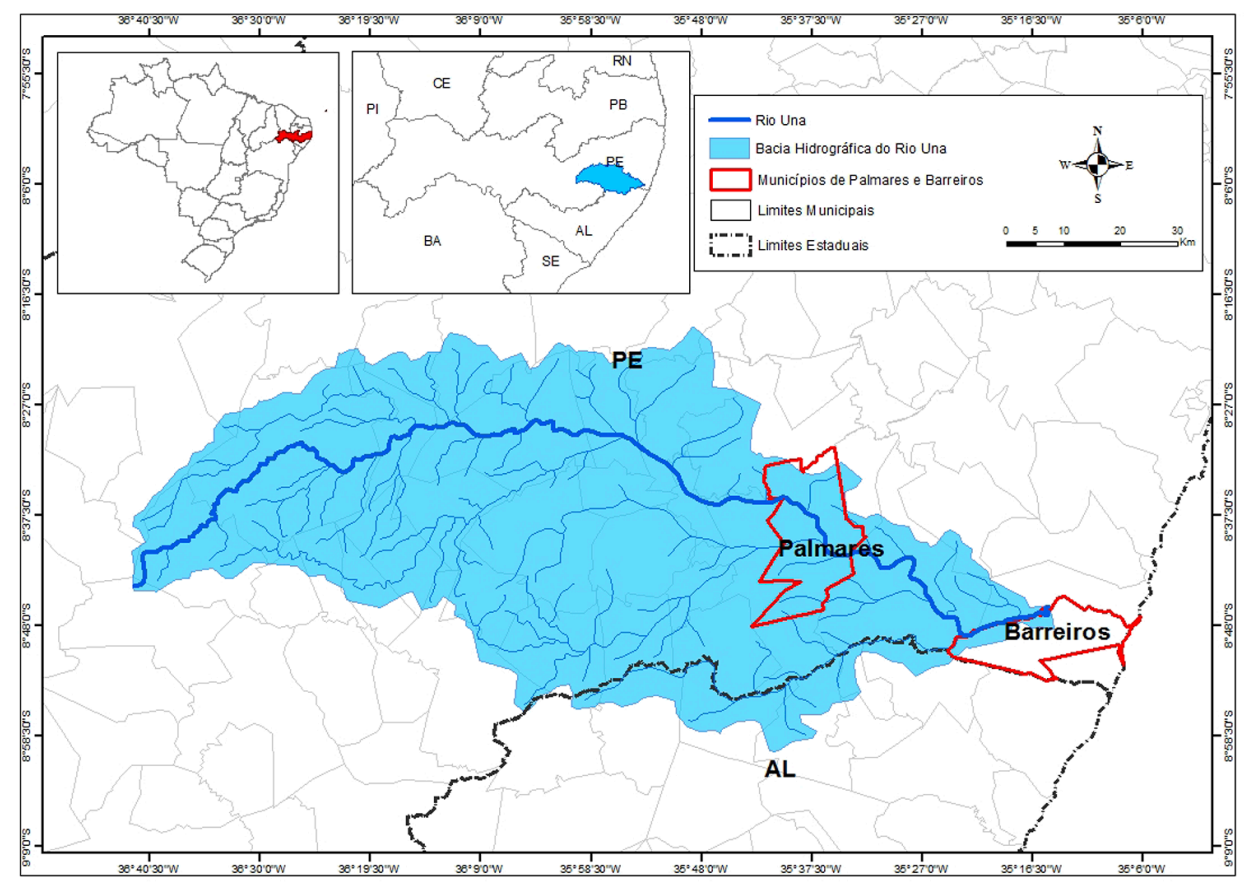

Fig. 1. Localización de la cuenca del río una, pernambuco / Brasil.

Fig. 1. Location basin river una, pernambuco / Brazil. 
sirvió para el análisis estadístico descriptivo, calculando la desviación estándar, el coeficiente de variación y la distribución de la precipitación promedio mensual.

El Índice de Anomalía de Lluvia (IAC) permitió identificar la variabilidad de las precipitaciones en condiciones normales de precipitación de la región estudiada (SILVA 2011). En el IAC se ha realizado mediante las siguientes ecuaciones: en busca de anomalías positivas superiores a los valores medios que se utilizan para la ecuación 1 , y negativo para las anomalías inferiores a los valores medios utilizados si la ecuación 2, con base en la metodología propuesta por ROOY (1965), adaptada por FREITAS (2004 y 2005).

$$
\mathrm{IAC}=3 \times\left[\frac{(N-\bar{N})}{(\bar{M}-\bar{N})}\right]
$$

$$
\mathrm{IAC}=-3 \times\left[\frac{(N-\bar{N})}{(\bar{X}-\bar{N})}\right]
$$

Donde, $\mathrm{N}=$ precipitación anual $(\mathrm{mm}), \bar{N}=$ precipitación media anual de la serie de 30 años (mm), $\bar{M}=$ promedio de los 10 casos de mayor precipitación anual de la serie $(\mathrm{mm})$, y $\bar{X}=$ promedio de 10 casos de menor precipitación de la series $(\mathrm{mm})$.

El IAC permite ordenar el volumen precipitado tanto en los años secos como húmedos de la siguiente manera:

$>4$ - extremadamente húmedo

2 a 4 - muy húmedo

0 a 2 - húmedo

0 a -2 - muy seco

$<-4$ - extremadamente seco

Para la identificación del sistema meteorológico que produjo el evento extremo, se utilizaron imágenes de satélite Meteosat de 17 y 18 de Junio de 2010.

La evaluación de los riesgos hidroclimático e impactos sociales se realizó través de trabajos de terreno en los municipios afectados, visitando específicamente las zonas próximas al río, donde vivían las familias cuyas viviendas fueron afectadas. Se entrevistó a los residentes más antiguos de la ciudad para obtener información sobre la ocurrencia de la inundación. Las diez entrevistas tuvo preguntas sobre: 1 . Datos personales; 2. La experiencia con las inundaciones; 3 . La principal causa de la inundación que se produjo en Junio de 2010; 4. Los impactos sociales de la inundación Junio de 2010.

\section{RESULTADOS}

Según el análisis estadístico se observó una marcada variabilidad en la serie temporal de las precipitaciones en Barreiros / PE, de acuerdo con la desviación estándar de los resultados $(548 \mathrm{~mm})$ y el coeficiente de variación $(26,3 \%)$. La distribución temporal de las precipitaciones, considerando el promedio mensual mostró que el período lluvioso se concentra en seis meses, de Marzo a Agosto. Las lluvias que se produjeron en 2010 presentaron desviaciones positivas de la media en los meses de enero y junio, con un $162 \%$ y $189 \%$ de la media, respectivamente.

El análisis de datos de la ciudad de Palmares presentó concentración de la precipitación media anual entre los meses de Marzo y Agosto. El volumen promedio de lluvia registrada en este período corresponde al $77 \%$ del volumen medio anual es de 1.508 $\mathrm{mm}$, con precipitación típica del sistema de lluvias del Este del Noreste, influenciado por la acción de las Ondas de Este, sobre todo durante el otoño y el invierno. Entre los meses más lluviosos se destacan Mayo, Junio y Julio, que tienen una media de $217 \mathrm{~mm}$ de lluvia, $268 \mathrm{~mm}$ y $246 \mathrm{~mm}$, respectivamente.

\section{Índice de Anomalía de lluvia}

En el análisis realizado con el IAC para la ciudad de Barreiros (Fig. 2), se observó 
valores positivos y negativos, lo que implica que determinado año puede ser lluvioso o seco. En función del resultado del índice, puede presentar categorías extremadamente húmedo a extremadamente seco. El año 2010 corresponde a la categoría de año seco (IAC -1,22). Por lo tanto, podemos afirmar que el año 2010, siendo considerado seco de acuerdo con el IAC, la precipitación en junio de este mismo año fue de tal intensidad y con desviaciones positivas de la media, que provocó impactos significativos para la población local.
Para la ciudad de Palmares (Fig. 3), los resultados presentaron gran variabilidad interanual de las precipitaciones. Se registró la ocurrencia de años secos y años húmedos, con valores que van desde $-6,2$ (1993) y 5,2 (en 1986). A partir de los cálculos de la IAC y la figura 3 , el año 2010 fue considerado como húmedo, no demostrando gran desviación anual (IAC 1,4), sin embargo presentó un gran desvío para el mes de Junio del mismo año.

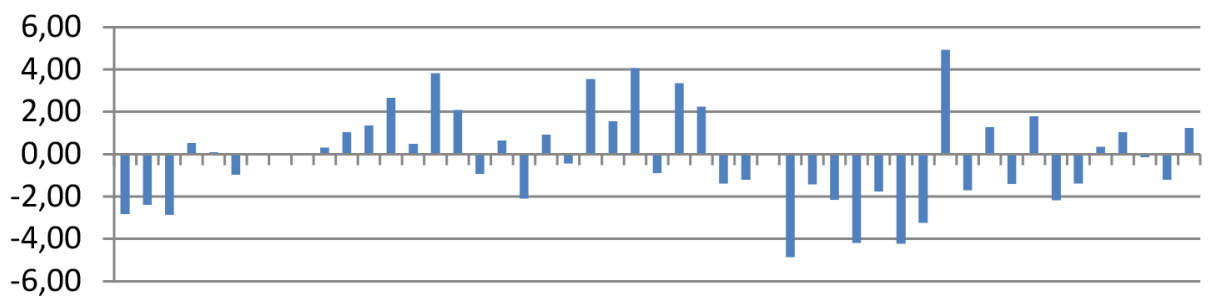

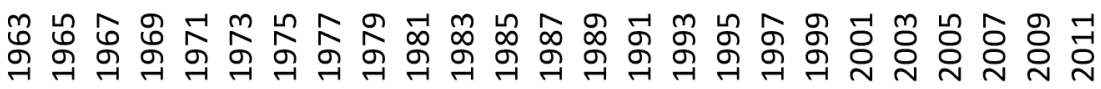

$\square$ IAC

Fig. 2. Indice de anomalía de lluvia para la ciudad de barreiros / Pe.

Fig. 2. Rainfall anomaly index for the city of barreiros / Pe.

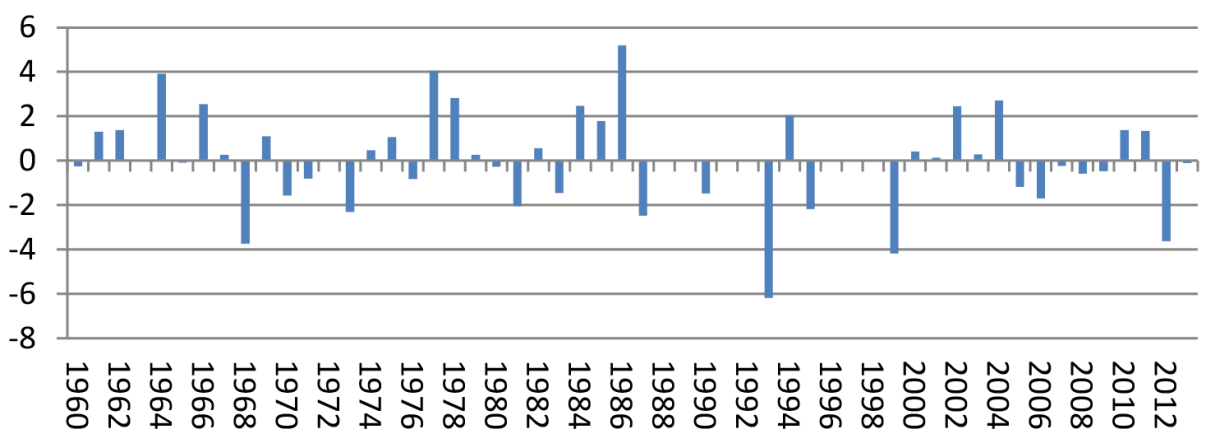

IAC

Fig. 3. Índice de anomalía de lluvia para la ciudad de palmares / Pe

Fig. 3. Rainfall anomaly index for the city of palmares / Pe. 
Entre el 16 y 18 de junio de 2010, un evento climático extremo llegó a la costa, alcanzando también la Zona da Mata y Agreste de los estados de Paraíba,
Pernambuco y Alagoas, causando grandes daños económicos y sociales (Fig. 4A, 4B, 4C, 4D).

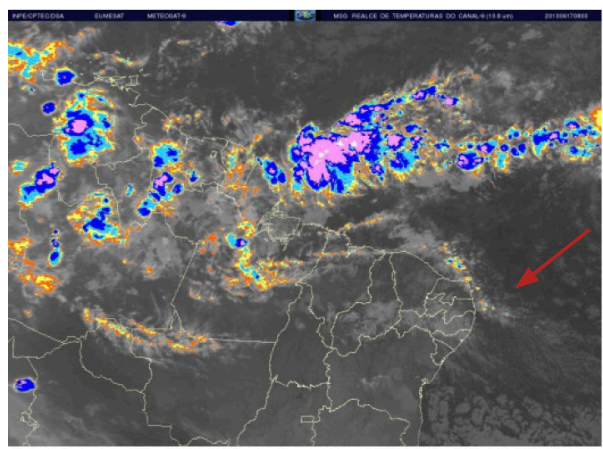

$(4 \mathrm{~A})$

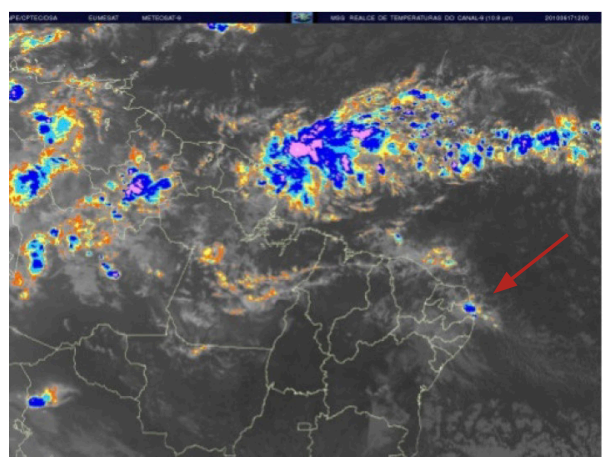

$(4 \mathrm{C})$

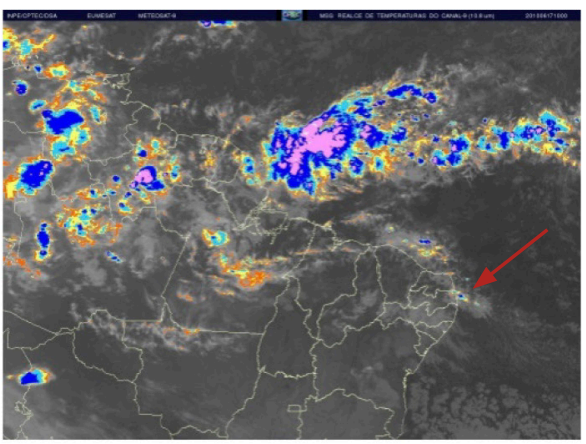

(4B)

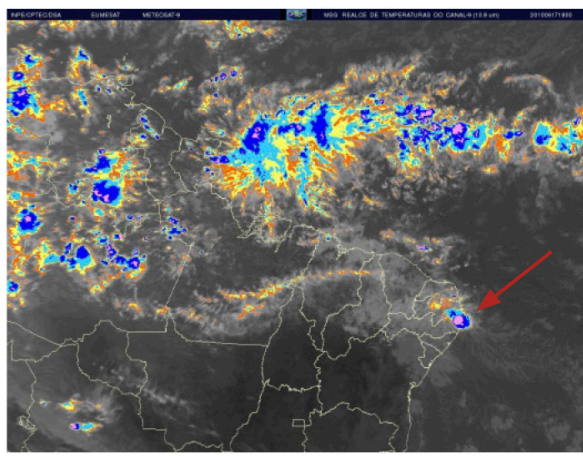

(4D)

Figs. 4A, 4B, 4C, 4D: imágenes de satélite meteosat - día 17.06.2010.

Figs. 4A, 4B, 4C, 4D: meteosat satellite images - day June 17, 2010.

De acuerdo con las figuras mencionadas se observa una Onda del Este intensificada por la proximidad de la ZCIT, que fue la responsable por la gran precipitación registrada en el período; las imágenes de satélite Meteosat de 17 de Junio muestran el movimiento de la Onda de Este.

\section{Riesgos hidroclimáticos e impactos sociales}

Se identificaron los riesgos en las áreas de estudio relacionados con los procesos climáticos e hidrológicos característicos de las zonas tropicales húmedas. Las precipitaciones extremas causadas por la Onda del Este en la costa oriental del Noreste, principalmente durante el otoño y el invierno en el hemisferio sur, aumentan el nivel de los ríos, que a su vez causan inundaciones. Estos procesos físicos cuando relacionados a la mala o inexistente planificación urbana, además de la desigualdad social intensa (factores que agravan la vulnerabilidad de las poblaciones), aumentan el riesgo de ocurrencia de desastres naturales en función de la probabilidad de ocurrencia de los sistemas meteorológicos de gran magnitud. 
Los residentes de Palmares contestaron no haber sido testigos de inundaciones con la misma proporción a las que ocurrieron en 2010 , con un volumen de agua superior al normal en comparación con las inundaciones anteriores. También informaron sobre las pérdidas económicas, de hogares y presencia de algunas enfermedades causadas por las inundaciones.

Debido a la intensa precipitación registrada en Junio de 2010 y el transborde del río Una, afectando los municipios de Barreiros y Palmares / PE, en inundaciones y también deslizamientos causando daño en residencias y comercios, pérdida de vidas humanas y urbana, (Fig. 5 y 6).

Los municipios de Barreiros y Palmares en el 2010 fueron fuertemente afectados por los desastres naturales relacionados con la lluvia. Normalmente, las inundaciones y aluviones causan severos daños materiales y dependiendo de su magnitud, graves daños humanos (ZAMPARONI 2012).

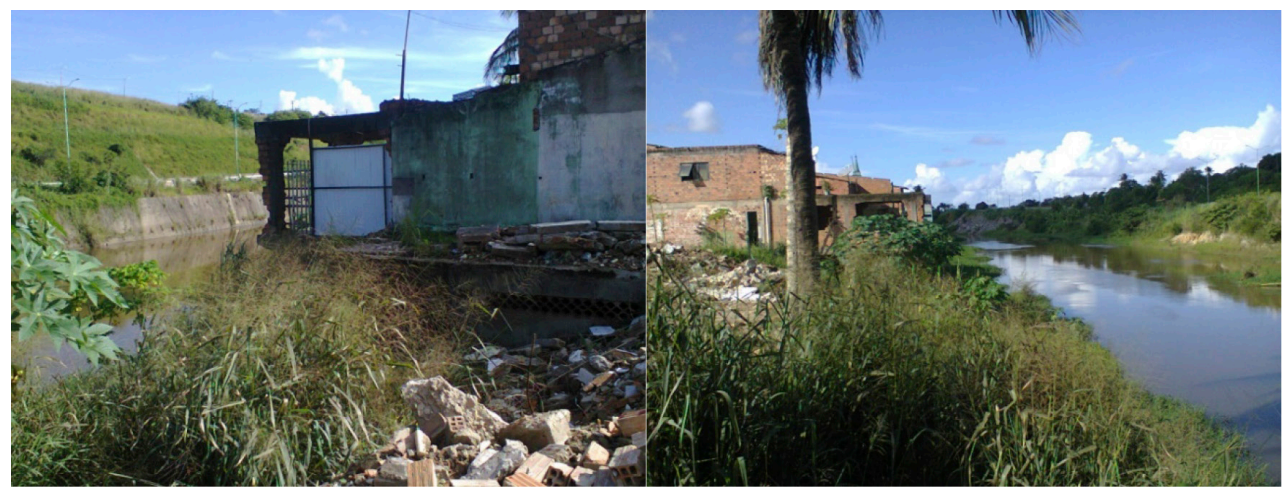

Figs. 5 y 6. Ejemplo de residencias afectadas por la inundación de 2010 en palmares / Pe (Mayo de 2014).

Figs. 5 y 6. Homes affected by the 2010 flood in palmares / Pe (May, 2014).

\section{CONCLUSIONES}

En la serie histórica de precipitaciones de Barreiros y Palmares se registró con más evidencia la variabilidad anual y mensual. En el análisis mensual, las mayores precipitaciones se concentran en los meses de Marzo a Agosto, siendo Mayo, Junio y Julio el trimestre más lluvioso. A través del análisis de las imágenes de satélite Meteosat, teniendo en cuenta los sistemas atmosféricos que operan en la región asociada a los elementos, se identificó la influencia de la Onda del Este, que actuó durante Junio de 2010 causando el desborde del río Una y las inundaciones en Barreiros y Palmares / PE.
Basado en el uso de Índice de Anomalía de lluvia (IAC) el año 2010, fue clasificado en la categoría seco en Barreiros y categoría húmedo en Palmares. Las fuertes lluvias concentradas en Junio con desviaciones positivas de la media fueron significativas y provocaron la inundación que causó graves daños a la población local.

Los riesgos observados en las áreas de estudio están asociados a los procesos de urbanización y a los eventos climáticos e hidrológicos característicos de las zonas tropicales húmedas. El evento de lluvia extrema modulada por la Onda del Este aumentó el nivel del río Una, causando inundaciones en las ciudades de Barreiros y 
Palmares. Estos procesos físicos asociados a la planificación urbana ineficiente y la intensa desigualdad social, agravó la vulnerabilidad de la población.

\section{AGRADECIMIENTOS}

FACEPE para promover esta investigación.

\section{REFERENCIAS}

BARBOSA, R. L, 2007. Interação das perturbações convectivas iniciadas na costa Norte do Brasil com distúrbios ondulatórios de leste. Dissertação (Mestrado em Meteorologia) - Instituto Nacional de Pesquisas Espaciais - INPE. São José dos Campos.

CASTRO, A. L. C, 2003. Manual de Desastres: desastres naturais. Brasília: Ministério da Integração Nacional.

CASTRO, C. M.; M. N. O. PEIXOTO \& G. A. P. RIO, 2005. Riscos ambientais e Geografia: conceito, ações, abordagens e escalas. Anuário do Instituto de Geociências, 28 (2): 11-30.

CIDADE, F. C. F., 2013. Urbanização, ambiente, risco e vulnerabilidade: em busca de uma construção interdisciplinar. Cad. Metrop, 15(29): 171-191.

FREITAS, M. A. S., 2004. A Previsão de Secas e a Gestão Hidroenergética: O Caso da Bacia do Rio Parnaíba no Nordeste do Brasil. In: Seminário Internacional sobre Represas y Operación de Embalses, 2004, Puerto Iguazú. Anais do Seminário Internacional sobre Represas y Operación de Embalses. Puerto Iguazú: CACIER, 1:1-1.

FREITAS, M. A. S., 2005. Um Sistema de Suporte à Decisão para o Monitoramento de Secas Meteorológicas em Regiões Semi-
Áridas. Revista Tecnologia, Fortaleza, 5: 84-95.

GIRÃO, O., 2012. Reconstrução do clima no Nordeste brasileiro: secas e enchentes do século XIX. Finisterra, 42(93): 29-47.

IBGE, INSTITUTO BRASILEIRO DE GEOGRAFIA E ESTATÍSTICA, 2014. www.ibge.gov.br.

IPCC. PAINEL INTERGOVERNAMENTAL SOBRE MUDANÇAS CLIMÁTICAS, 2014. Organización Meteorológica Mundial. Organismo especializado de las Naciones Unidas. Comunicado de prensa. No 987.

JACOBI, P., 2004. Impactos socioambientais urbanos - do risco à busca da sustentabilidade. In: MACHADO, C. C. C.; R. S. NÓBREGA; T. H. OLIVEIRA, \& K. M. A. S. ALVES, 2012. Distúrbio Ondulatório de Leste como condicionante a eventos extremos de precipitação em Pernambuco. Revista Brasileira de Climatologia, 11.

MARENGO, J. A., 2008. Vulnerabilidade, Impactos e Adaptação à Mudanças do Clima no Semi-árido do Brasil, Brasília.

MENDONÇA, F. (org.), 2004. Impactos socioambientais urbanos. Curitiba, PR: Editora UFPR.

MENDONÇA, F., 2010. Riscos e vulnerabilidades socioambientais urbanos a contingência climática. Mercator, 9 (1).

MOLLION, L.C.B.\&S.O.BERNARDO, 2002. Uma revisão da dinâmica das chuvas no Nordeste brasileiro. Revista Brasileira de Meteorologia, 17 (1): 1-10.

MONTEIRO, C. A. F., 1996. Os Geossistemas como Elemento de Integração 
na Síntese Geográfica e Fator de Promoção Interdisciplinar na Compreensão do ambiente. Revista de Ciências Humanas Florianópolis, 14 (19): 67-101.

MOURA, R. \& L. A. SILVA, 2008. Desastres naturais ou negligência humana? Revista Geografar, 3 (1): 58-72.

NEIVA, E. J. F., 1975. An investigation of wave-type disturbances over the Tropical South Atlantic. Dissertação (Mestrado em Meteorologia) - Naval Post Graduate School, Monterrey, California, USA.

ROOY, M. P. VAN, 1965. A rainfall anomaly index independent of time and space. Notes, 14: 43.

SANTOS, A. H. M.; M. R. DA S. ARAGÃO; M. DE F. CORREIA; H. A. DE ARAUJO \& A. B. SILVA, 2012. Distúrbio Ondulatório de Leste e seus impactos na cidade de Salvador. Revista Brasileira de Meteorologia, 27 (3): 355 364.

SILVA, D. F., 2011. Influência da variabilidade climática e da associação de fenômenos climáticos sobre sub-bacias do rio São Francisco. Revista Brasileira de Ciências Ambientais, 19.
SILVEIRA, R. D. \& M. DA G. B. SARTORI, 2010. Relação entre tipos de tempo, eventos de precipitação extrema e inundações no espaço urbano de São Sepé, RS. Revista Brasileira de Climatologia, 7 (6).

TOMINAGA, L. K., J. SANTORO, \& R. AMARAL, 2009. Desastres Naturais: conhecer para Prevenir. São Paulo: Instituto Geológico.

VEYRET, Y. (Org.), 2007. Os riscos: o homem como agressor e vítima do meio ambiente. São Paulo: Contexto.

YAMAZAKI, Y. \& V.B. RAO, 1977. Tropical cloudiness over South Atlantic Ocean. J. Meteor. Soc. Japan, 55 (2): 205207.

ZAMPARONI, C. A. G. P., 2012. Riscos e desastres naturais em ambiente urbano: o exemplo de Cuiabá/MT. Revista Brasileira de Climatologia, 10. 